\title{
Editorial
}

Alan N. Sandler MSc MBCHB FRCPC, Roger Schmid MD, ${ }^{\dagger}$

Joel Katz $\mathrm{PhD}^{\ddagger}$

\section{Epidural ketamine for postoperative analgesia}

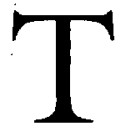

WO factors have increased interest in ketamine for postoperative analgesia. The first was the discovery of the N-Methyl-D-aspartate (NMDA) receptor and its role in central pain processing and spinal cord neural plasticity. ${ }^{1}$ Ketamine is one of two clinically useful NMDA receptor antagonists available (the other is dextromethorphan). Ketamine binds non-competitively to the PCI, (phencyclidine) recognition site in the NMDA receptor channel. ${ }^{2}$ In addition, interest in the concept of precmptive analgesia makes ketamine a natural candidate for investigation of postoperative pain relief since blockade of the NMDA receptor reduces noxious stimulusinduced allodynia and hyperalgesia. To avoid the well-known psychomimetic effects of ketamine, investigators have focused on the use of smaller doses than are required for general anaesthesia and alternative administration routes for postoperative analgesia. Spinal administration would seem to be especially interesting due to the proximity of the NMDA receptors and the potential for decrease in dose requirements. Reports on intraspinal administration of ketamine include caudal subarachnoid ketamine administration, with or without local anaesthetic agents, lumbar epidural ketamine alone or with local anaesthetic agents and/or opioids and thoracic epidural ketamine with opioids. There are several case reports, comments and uncontrolled studies related to epidural ketamine analgesia ${ }^{3-5}$ but only a few controlled clinical trials. ${ }^{6-8}$ Although two observational studies claimed epidural ketamine is effective as a postoperative analgesi $i^{3,4}$ other uncontrolled trials could find little or no postoperative analgesic effect. ${ }^{5}$ Similarly, properly controlled trials using either small (4-8 $\mathrm{mg})^{7}$ or large $(30 \mathrm{mg})^{6,8}$ doses were unable to document effective postoperative epidural analgesia. Epidural ketamine may have an adjuvant effect when added to epidural morphine ${ }^{8}$ or local anaesthetic agents. ${ }^{9}$ Wong et al.$^{8}$ administered $10 \mathrm{mg}$ ketamine and $0.5 \mathrm{mg}$ morphine epidurally to patients scheduled for major joint replacement. The kelamine/morphine combination produced the same degree of analgesia (with movement) as did $2 \mathrm{mg}$ epidural morphine alone. In this study $10 \%$ of patients displayed psychomimetic effects requiring treatment. Yanli et al., ${ }^{9}$ in a controlled study, added $25 \mathrm{mg}$ ketamine to a bupivacaine $0.5 \%$ with 1;200,000 adrenalin mixture and administered $20 \mathrm{ml}$ epidurally to patients undergoing lower abdominal or orthopaedic surgery. There was a small but significant decrease in onset time to anaesthesia and a slightly higher segmental blockade in the ketamine group. There were no difference between the ketamine group and the non-ketamine group with regard to postoperative analgesic requirements and no adverse psychomimetic effects were seen. There are some claims that epidural ketamine and morphine combinations may have a preemptive analgesic effect. ${ }^{10,11}$ Choe et al. ${ }^{11}$ in a controlled study added a large close of ketamine $(60 \mathrm{mg}$ ) to $2 \mathrm{mg}$ epidural morphine and administered the combination before induction of general anaesthesia or 2-3 hr after the start of abdominal surgery. The only measure of analgesic efficacy was analgesic duration which was significantly prolonged in the preincisional group. No psychomimetic effects were noted. The comparative roles of the ketamine or the morphine in the possible preemptive effect are unclear. Wong et al. ${ }^{10}$ used a smaller dose of ketamine $(20 \mathrm{mg})$ with $1.5 \mathrm{mg}$ morphine given epidurally before or after incision to parients having knee joint replacement. Both groups received epidural anaesthesia with lidocaine intraoperatively. Postoperatively all patients received epidural ketamine $(10 \mathrm{mg}$ ) and morphine $(1 \mathrm{mg})$ in lidocaine every $12 \mathrm{hr}$ and also had additional PCA morphine available for rescue analgesia. Administration of the morphine/ketamine/lidocaine mixture before surgery resulted in less pain and lower PCA requirements than administration $30 \mathrm{~min}$ after surgery had commenced. The preemptive effect of epidural ketamine alone is difficult to evaluate in this study as com-

"Department of Anaesthesia, Toronto Hospital, and Mt. Sinai Hospital, University of Toronto, Toronto, Ontario.

tAnaesthesiologische Universitaetsklinik, Frciburg, Germany.

†Department of Public Health Sciences and Anaesthesia, University of Toronto. 
binations of epidural agents were always used. Spinal (epidural and subarachnoid) administration of ketamine should continue to be approached with caution. Although animal studies have demonstrated the relative safety of spinal ketamine without preservative ${ }^{12}$ doserelated neuro-toxicity studies have not been performed. Most of the studies do not provide good data concerning psychomimetic side effects and this knowledge is important especially in studies evaluating larger bolus doses, continuous infusions or prolonged administration in the postoperative period. Pharmacokinetic studies in humans after a single $5 \mathrm{mg}$ epidural dose indicated rapid access to the circulation from the epidural space. ${ }^{13}$ There are no circulatory or CSF pharmacokinetic data from human clinical trials with epidural ketamine.

In this issue Abdel-Ghaffar et al., in a placebocontrolled, randomised blinded trial administered $30 \mathrm{mg}$ epidural ketamine prior to the induction of anaesthesia or $20 \mathrm{~min}$ after skin incision to women undergoing abdominal hysterectomy. They found that epidural ketamine reduced post-hysterectomy pain on the basis of prolongation of time to first request for postoperative analgesic and reduction in postoperative epidural PCA requirements. These effects were not dependent on the timing of the epidural ketamine administration i.e., improved analgesia was present to the same degree in both the pre- and post-incision epidural ketamine groups.

Thus, evidence is accumulating that epidural ketamine, although not a potent epidural analgesic alone, may have an additive effect with other well known epidural analgesic agents i.e., opioids or local anaesthetic drugs. Although statistically significant, the analgesic effects of epidural ketamine are relatively small and continue to point to combination therapies with their additive and synergistic properties as the desired model for postoperative analgesia.

\section{Kétamine péridurale pour l'analgésie postopératoire}

Deux facteurs ont accru l'intérêt envers la kétamine pour l'analgésie postopératoire. Le premier a été la découverte du récepteur N-méthyl-D-aspartate (NMDA) et de son rôle dans le traitement central de la douleur et dans la plasticité neurale de la moelle ${ }^{1}$. La kétamine est l'un des deux antagonistes du récepteur NMDA cliniquement intéressants, l'autre étant le dextrométhorphan. La kéta- mine se lie de façon non compétitive au site de reconnaissance PCI (phencyclidine) dans le canal du récepteur NMDA ${ }^{2}$. En outre, l'intérêt pour le concept d'analgésie préemptive fait de la kétamine un candidat naturel dans la recherche sur le soulagement de la douleur postopératoire puisque le blocage du récepteur NMDA réduit l'allodynie et l'hyperalgésie induites par un stimulus. Pour éviter les effets psychotomimétiques bien connus de la kétamine, les chercheurs ont concentré leurs efforts sur l'utilisation de doses plus faibles que celles requises pour l'anesthésie générale de même que sur des voies d'administration différentes pour l'analgésie postopératoire. L'administration rachidienne apparaîtrait particulièrement intéressante à cause de la proximité des récepteurs NMDA et du potentiel de réduction des doses requises. Les études concernant l'administration rachidienne de kétamine incluent l'administration subarachnoïdienne par voie caudale, avec ou sans anesthésiques locaux, la kétamine péridurale lombaire seule ou avec anesthésiques locaux et/ou opiacés et la kétamine par voie péridurale thoracique avec opiacés. Il existe plusieurs études de cas, commentaires et études non contrôlées ayant trait à l'analgésie péridurale par la kétamine $^{3-5}$ mais seulement quelques études cliniques bien contrôlées ${ }^{6,8}$. Bien que deux études descriptives prétendent que la kétamine péridurale est efficace comme analgésique postopératoire ${ }^{3,4}$, d'autres études non contrôlées n'ont pu mettre en évidence d'effet analgésique postopératoire significatif. De la même façon, des études bien contrôlées, utilisant ou de petites doses $(4-8 \mathrm{mg})^{7}$ ou des plus larges $(30 \mathrm{mg})^{6,8}$ ont été incapables de mettre en évidence une analgésic péridurale postopératoire réelle. La kétamine péridurale peut avoir un effet adjuvant lorsque ajouté à la morphine péridurale ${ }^{8}$ ou à des agents anesthésiques locaux ${ }^{9}$. Wong et al. ${ }^{8}$ ont administré $10 \mathrm{mg}$ de kétamine et $0,5 \mathrm{mg}$ de morphine par voie péridurale à des patients devant subir un remplacement d'une articulation majeure. La combinaison kétamine/morphine a produit le même degré d'analgésie (avec mobilisation) que celle produite par $2 \mathrm{mg}$ de morphine seule par voie péridurale. Dans cette étude, $10 \%$ des parients ont présenté des effets psychotomimétiques nécessitant traitement. Yanli $e t a l^{9}$, dans une étude contrôlée, ont ajouté $25 \mathrm{mg}$ de kétamine à une solution de bupivacaïne 0,5\% adrénalinée à 1:200,000 et ont administré par voie épidurale $20 \mathrm{ml}$ de la solution à des patients pour des chirurgies abdominales basses ou orthopédiques. Le groupe kétamine a présenté un début d'anesthésie légèrement mais significativement plus rapide et un bloc segmentaire légèrement plus haut. Il n'y avait pas de différence entre les deux groupes, avec ou sans kétamine, quant aux besoins analgésiques postopératoires et on n'observa pas d'effets psy- 
chotomimétiques indésirables. Certains prétendent que les combinaisons péridurales de kétamine et de morphine ont un effet analgésique de type préemptif ${ }^{10,11}$. Choe et al. ${ }^{11}$, dans une étude contrôlée, ont ajouté une dose élevée de kétamine $(60 \mathrm{mg})$ à $2 \mathrm{mg}$ de morphine péridurale et ont administré cette combinaison avant l'induction de l'anesthésie générale ou 2 à 3 heures après le début de la chirurgie abdominale. La seule mesure de l'effet analgésique était la durée de l'analgésie, et cette dernière était significativement prolongée dans le groupe pré-incision. On n'a pas rapporté d'effets psychotomimétiques, mais les rôles comparatifs de la kétamine ou de la morphine dans un effet préemptif possible ne sont pas clairs. Wong et al ${ }^{10}$ ont utilisé une dose plus faible de kétamine $(20 \mathrm{mg}$ ) associée à $1,5 \mathrm{mg}$ de morphine et l'ont administré avant ou après l'incision chez des patients subissant une chirurgie de remplacement du genou. Les deux groupes ont reçu une anesthésie péridurale avec lidocaine durant l'intervention. Après l'opération, tous les patients ont reçu, à toutes les 12 heures, par voie péridurale, $10 \mathrm{mg}$ de kétamine et $1 \mathrm{mg}$ de morphine diluées dans la lidocaïne, et ils avaient accès à une ACP à la morphine pour analgésie de sauvetage. L'administration de la mixture morphine/kétamine/ lidocaiine avant la chirurgie a entraîné moins de douleur postopératoire et moins d'utilisation de l'ACP que l'administration 30 minutes après le début de la chirurgie. Dans cette étude, l'effet préemptif de la kétamine péridural est difficile à cerner puisque des combinaisons de médicaments par voie péridurale ont toujours été utilisées. L'administration de kétamine par voie péridurale ou sous-arachnoïdienne devrait continuer à se faire avec circonspection. Même si les études animales ont démontré la sécurité relative de la kétamine sans agent de conservation ${ }^{12}$, les études de neuro-toxicité reliées à la dose n'ont pas été réalisées. La plupart des études ne fournissent pas de données solides concernant les effets secondaires psychotomimétiques et cette information devient importante surtout pour évaluer des bolus plus considérables, des infusions continues ou des administrations prolongées en période postopératoire. Des études pharmacocinétiques chez des humains après une dose épidurale unique de $5 \mathrm{mg}$ ont montré un accès rapide à la circulation à partir de l'espace épidural ${ }^{13}$. Il n'y a pas de données pharmacocinétiques concernant la circulation ou le LCR à partir d'études cliniques humaines de la kétamine péridurale.

Dans ce numéro du Journal, Abdel-Ghaffar et al., dans une étude à l'aveugle, aléatoire et contrôlée par placebo, ont administré à des patientes subissant une hystérectomie abdominale $30 \mathrm{mg}$ de kétamine par voie péridurale avant l'induction de l'anesthésie ou $20 \mathrm{~min}$ utes après l'incision cutanée. Ils ont constaté que la kéta- mine par voie péridurale réduisait la douleur post hystérectomie si l'on se fie à l'allongement du temps écoulé avant la première demande d'analgésiques et à la réduction des besoins d'ACP péridurale. Ces effets ne dépendaient pas du moment de l'administration de la kétamine i.e., l'amélioration de l'analgésie était présente dans les deux groupes kétamine.

Donc, les preuves s'accumulent concernant la kétamine qui, bien que n'étant pas un analgésique puissant en soi par voie péridurale, peut avoir un effet additif avec plusieurs agents analgésiques périduraux bien connus i.e., les opiacés et les anesthésiques locaux. Même s'ils sont statistiquement significatifs, les effets analgésiques de la kétamine par voie péridurale sont relativement discrets et continuent à indiquer que les thérapies de combinaison exploitant les propriétés additives ou synergiques des divers agents sont le modèle désiré pour l'analgésie postopératoire.

\section{References}

1 Woolf CJ, Thompson SWN. The induction and maintenance of central sensitization is dependent on $\mathrm{N}$-methyl $D$-aspartic acid receptor activation; implications for the treatment of post-injury pain hypersensitivity states. Pain 1991; 44; 293-9.

2 Foster AC, Fugg GE. Neurobiology. Taking apart NMDA receptors. Nature 1987; 329: 395-6.

3 Islas J-A, Astorga J, Laredo $M$. Epidural ketamine for control of postoperative pain. Anesth Analg 1985; 64: 1161-2.

4 Naguib $M$, Adu-Gyamfi $\Upsilon$, Absood GH, Farag $H$, Gyasi $H K$. Epidural ketamine for postoperative analgesia. Can Anaesth Soc J 1986; 33; 16-21.

5 Ravat F, Dorne R, Baechle JP, et al. Epidural ketamine or morphine for postoperative analgesia. Anesthesiology 1987; 66: 819-22.

6 Peat ST, Bras P, Hanna MH. A double-blind comparison of epidural ketamine and diamorphine for postoperative analgesia. Anaesthesia 1989; 44: 555-8.

7 Kawana $\Upsilon$, Sato $H$, Shimada $H$, et al. Epidural ketamine for postoperative pain relief after gynecologic operations: a double-blind study and comparison with epidural morphine. Anesth Analg 1987; 66: 735-8.

8 Wong C-S, Liaw W-J, Tung C-S, Su Y-F, Ho S-T. Ketamine potentiates analgesic effect of morphine in postoperative epidural pain control. Reg Anesth 1996; 21: 534-41.

9 Tanli $\Upsilon$, Eren $A$. The effect of extradural ketamine on onset time and sensory block in extradural anaesthesia with bupivacaine. Anaesthesia 1996; 51: 84-6.

10 Wong C-S, Lu C-C, Cherng C-H, Ho S-T. Pre-emptive analgesia with ketamine, morphine and epidural lido- 
caine prior to rotal knee replacement. Can J Anaesth 1997; 44: 31-7.

11 Choe $H$, Choi $Y \cdot S$, Kim $Y-H$, at al. Epidural morphine plus ketamine for upper abdominal surgery: improved analgesia from preincisional versus postincisional administration. Anesth Analg 1997; 84: 560-3.

12 Malinopsky J-M, Lepage J-Y, Cozian A, Mussini J-M, Pinaudt $M$, Sourom $R$. Is ketamine or its preservative responsible for neurotoxicity in the rabbit? Anesthesiology 1993; 78: 109-13.

13 Pedraz $I$, Lanao JM, Calvo MB, Muriel $C$, HernandezArbeiza J, Dominguez-Gil A. Pharmacokinetic and clinical evaluation of ketamine administered by i.v. and 